\title{
Identification and biological activity of ovine and caprine calcitonin receptor-stimulating peptides 1 and 2
}

\author{
Christopher J Charles, Takeshi Katafuchi ${ }^{1}$, Timothy G Yandle and Naoto Minamino ${ }^{\mathbf{1}}$ \\ Christchurch Cardioendocrine Research Group, University of Otago Christchurch, Christchurch 8140, New Zealand \\ ${ }^{1}$ National Cardiovascular Center Research Institute, 5-7-1 Fujishirodai, Suita, Osaka 565-8565, Japan \\ (Correspondence should be addressed to N Minamino; Email: minamino@ri.ncvc.go.jp)
}

\begin{abstract}
We have recently reported the isolation of three new members of the calcitonin (CT) gene-related peptide family of peptides, the CT receptor (CT-R)-stimulating peptides (CRSPs). We now report the sequencing and characterization of ovine/caprine CRSP-1 and caprine CRSP-2. Mature ovine and caprine CRSP-1 are identical and have strong structural homology to CRSP-1s identified to date from other species. As with other CRSP-1s, ovine/caprine CRSP-1 binds to and activates the CT-R but not the CT-like receptor (CL-R) in combination with the receptor activity-modifying proteins (RAMPs). By contrast, caprine CRSP-2 does not activate any of these receptor-RAMP complexes. Intravenous infusions of ovine CRSP-1 to normal conscious sheep induced dose-dependent reduction in plasma total $\mathrm{Ca}$ levels $(P=0 \cdot 02)$ and corrected $C$ a levels $(P=0 \cdot 017)$ associated with increases in plasma cAMP $(P=0 \cdot 002)$. CRSP-1 reduced both
\end{abstract}

plasma amino-terminal pro-C-type natriuretic peptide levels $(P=0 \cdot 006)$ and plasma renin activity $(P=0 \cdot 028)$. There were no significant effects observed on hemodynamic or renal indices measured. In conclusion, we have sequenced ovine/caprine CRSP-1 and caprine CRSP-2 precursors. This newly identified CRSP-1 has been shown to share the structural and biological features of CRSP-1s known to date. In vivo studies confirm that ovine CRSP-1 reduces plasma $\mathrm{Ca}$ levels in sheep, presumably via a cAMP-mediated mechanism. By contrast, caprine CRSP-2 did not stimulate any combination of CT-R, CL-R, and RAMPs. Accession numbers of cDNA determined in this study are caprine CRSP-1, AB364646; caprine CRSP-2, AB364647; and ovine CRSP-1, AB364648.

Journal of Endocrinology (2008) 198, 429-437

\section{Introduction}

We have recently reported isolation of a novel peptide, calcitonin (CT) receptor-stimulating peptide (CRSP-1), from porcine brain extracts by monitoring cAMP release from the kidney epithelial cell line LLC-PK 1 (Katafuchi et al. 2003a). We have since identified two additional closely related peptides CRSP-2 and CRSP-3 also from porcine brain (Katafuchi et al. 2003b) and have also identified homologous peptides from cattle, dogs, and horse (Katafuchi et al. 2004). Each of these peptides shows sequence homology to CT and CT gene-related peptide (CGRP) and thus they make up a part of the CGRP family. Both mRNA expression and immunoreactive peptide content for CRSP-1 is widely expressed throughout the central nervous system and periphery with particularly high levels observed in the midbrain, hypothalamus, pituitary, and thyroid (Katafuchi et al. 2003a). CRSP-1 is a specific ligand for the CT receptor (CT-R) with porcine CRSP-1 evoking a 100-fold greater stimulation of cAMP from recombinant CT-R than porcine CT itself (Katafuchi et al. 2003a). By contrast, despite significant sequence homology to CGRP and adrenomedullin (AM), another member of the CGRP family, the CRSPs, appear not to be ligands for any of the combinations of the CT-like receptor (CL-R) and the three receptor activitymodifying proteins (RAMP1-3), namely the CGRP and AM receptors (Katafuchi et al. 2003a,b, 2004).

To date, there have been limited studies examining the bioactivity of CRSP-1 in vivo. Central administration of porcine CRSP-1 to rats suppressed food intake, increased body temperature, and decreased locomotor activity (Sawada et al. 2006). Systemic administration of porcine CRSP-1 in anaesthetized rats induced no significant change in arterial pressure but did induce a significant reduction in plasma Ca (Katafuchi et al. 2003a). There have been no in vivo studies of the biological actions of a CRSP-1 peptide administered to the species of origin. Accordingly, we here report the identification and in vitro characterization of ovine/ caprine CRSP-1 and caprine CRSP-2. Furthermore, we have examined the integrative hemodynamic, hormonal, electrolyte, and renal effects of ovine CRSP-1 in normal conscious sheep. 


\section{Materials and Methods}

\section{Isolation of caprine and ovine CRSP cDNAs}

Total RNAs were prepared from ovine thyroid gland and caprine hypothalamus using RNA-Bee reagent, and poly $\left(\mathrm{A}^{+}\right)$ RNA was purified from the RNAs using an mRNA purification system (Takara, Shiga, Japan). Libraries were constructed from cDNAs synthesized from $3 \mu \mathrm{g}$ poly $\left(\mathrm{A}^{+}\right)$ RNAs using TimeSaver cDNA synthesis kit according to the manufacturer's protocol. Approximately, $3 \times 10^{5}$ independent clones were screened by the standard protocol using ${ }^{32} \mathrm{P}-l$ labeled porcine full-length CRSP-1-cDNA. Hybridization and washing were performed at $42^{\circ} \mathrm{C}$ in $50 \%$ formamide, $6 \times$ SSC $(\mathrm{pH} 7 \cdot 0), 5 \times$ Denhardt's solution and $0.5 \% \mathrm{SDS}$, and $65^{\circ} \mathrm{C}$ in $0 \cdot 5 \times$ SSC and $0 \cdot 1 \%$ SDS respectively. The isolated positive clones were rescued as pBluescript SK- using Escherichia coli strain SOLR and helper phage. DNA sequences of these clones were determined by the dideoxynucleotide chain termination method using a DNA sequencer (310 Genetic Analyzer; Applied Biosystems, Foster City, CA, USA).

\section{Peptide synthesis}

Ovine/caprine CRSP-1 and caprine CRSP-2 were synthesized on an Abacus peptide synthesizer (Sigma Genosys) using Fmoc ( $\mathrm{N}$-(9-fluorenyl) methoxycarbonyl) strategy, purified by reversed phase HPLC. Correct synthesis of the peptides was verified by mass spectrometry and amino acid analysis, and their purity (more than 90\%) was confirmed on a separate HPLC system.

In vitro studies: measurement of $c$ AMP production

COS-7 cells were maintained in Dulbecco's Modified Eagle's Medium (DMEM) containing $100 \mu \mathrm{g} / \mathrm{ml}$ penicillin, $100 \mathrm{U} / \mathrm{ml}$ streptomycin, and 10\% BSA (FBS) in a humidified atmosphere of $95 \%$ air and $5 \% \mathrm{CO}_{2}$ at $37^{\circ} \mathrm{C}$. cDNAs of porcine CT-R, CL-R, and three RAMPs were ligated to a pcDNA3.1 mammalian expression vector as reported previously (Katafuchi et al. 2003a). Each combination of cDNAs of CT-R or CL-R, and one of three RAMPs was mixed at a ratio of 1:3. Transfection of mixed cDNAs was performed using Lipofectamine LTX with Plus reagent according to the manufacturer's protocol (Invitrogen). Following the transfection and further incubation for $24 \mathrm{~h}$, the cells were washed twice with DMEM plus $20 \mathrm{mM}$ HEPES (DMEM/HEPES ( $\mathrm{pH} 7 \cdot 4)$ ) and incubated with DMEM/HEPES plus 0.5 mM 3-isobutyl-1-methyl xanthine (IBMX). Following a 15-min incubation, the medium was replaced into DMEM/HEPES plus IBMX containing $0.05 \%$ BSA and a peptide of interest, and further incubated for $30 \mathrm{~min}$. Then, the incubation medium was collected, and an aliquot of it was succinylated, evaporated, and submitted to RIA for cAMP, as described previously (Katafuchi et al. 2003a).
Bioactivity of ovine CRSP-1 in conscious sheep

The Animal Ethics Committee of the University of Otago, Christchurch approved the study protocol. Six Coopworth ewes weighing 52-66 kg (Lincoln University Farm, Christchurch, New Zealand) were housed in an airconditioned light-controlled room and received a diet of lucerne chaff and food pellets providing $75 \mathrm{mmol}$ sodium and $150 \mathrm{mmol}$ potassium per day. Under general anesthesia (induced by $17 \mathrm{mg} / \mathrm{kg}$ thiopentone sodium and maintained by a mixture of halothane, nitrous oxide, and oxygen), a carotid artery was cannulated (16 G Angiocath; Becton Dickinson, Sandy, UT, USA) for direct measurement of arterial pressure and heart rate. Polyethylene catheters were placed in the jugular veins for blood sampling and measurement of right atrial pressure (RAP), and a SwanGanz thermodilution catheter (Edwards Life Sciences, Irvine, CA, USA) was placed in the pulmonary artery via the jugular vein for measurements of cardiac output. A Foley catheter was placed per urethra in the bladder to allow continuous collection of urine. The animals recovered for at least 7 days before experiments.

Each animal was studied on two occasions 2 days apart receiving vehicle control (0.9\% saline) and ovine CRSP-1. CRSP-1 was administered in incremental doses as i.v. infusions of $5 \mathrm{pmol} / \mathrm{kg}$ per min followed immediately by $50 \mathrm{pmol} / \mathrm{kg}$ per min each, for 60-min duration. Control and CRSP-1 were administered in a balanced, random-order design.

Arterial pressure and RAP were recorded using an in-house online data acquisition system, commencing $30 \mathrm{~min}$ before infusions and continuing for $60 \mathrm{~min}$ beyond end of the highest dose infusion. Heart rate and pressures were digitally integrated in 5-min recording periods and data recorded at preset intervals throughout the study. Cardiac output (thermodilution) was measured in triplicate (three values within 10\%) at preset intervals for the duration of infusions. Calculated total peripheral resistance was calculated as mean arterial pressure (MAP) divided by cardiac output.

Venous blood was drawn at preset intervals during the study protocol. Blood was taken into chilled EDTA tubes, centrifuged, and the plasma stored at $-80^{\circ} \mathrm{C}$ before assay for aldosterone (Lun et al. 1983), catecholamines (Goldstein et al. 1981), cortisol (Lewis et al. 1992), plasma renin activity (PRA) (Dunn \& Espiner 1976), amino-terminal pro-C-type natriuretic peptide (NT-CNP) (Prickett et al. 2001), and cAMP by commercially available kit (Biotrak, Amersham). Hematocrit was measured with each blood sample. Additional plasma samples were drawn into heparin tubes for routine biochemical analysis of calcium, sodium, potassium, creatinine, and albumin.

\section{Statistical analysis}

Results are expressed as the mean \pm s.E.M. Two-way ANOVA with time as a repeated measure was used to determine time and treatment differences between CRSP-1 and control arms 
A AGTGCCATCGCCTGCCGCCAAAGCTACTGCTGCTGCTCCTTCCTCTGCTCCCAGCCACCT GGTACCGGCTGTCAGAGAGGTGTCATGGGGTTCTGGAAGTTCCCCCCATTCTTGGTCCTC $\begin{array}{lllllllllllllll}M & G & F & W & K & F & P & P & F & L & V & L\end{array}$ AGCATCCTGGTCTTGTACCAGGCAGGCATGTTTCATGCAGCACCATTCAGGTCTGTCTTT $\begin{array}{lllllllllllllllllllll}S & I & L & V & L & Y & Q & A & G & M & F & H & A & A & \mathrm{P} & \mathrm{F} & \mathrm{R} & \mathrm{S} & \mathrm{V} & \mathrm{F}\end{array}$ GATGGGCGTTTTGATCCTGCTACCCTGGATGAGGAGGAATCGCGCCTCCTACTGGCTGCG $\begin{array}{llllllllllllllllllll}D & G & R & F & D & P & A & T & L & D & E & E & E & S & R & L & L & L & A & A\end{array}$ ATGGTGAATGACTACGAGCAGATGAGGACCCGGGAGTCGGAGAAGGCTCAGAAGACCGAG

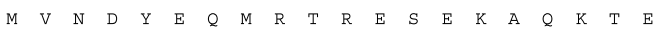
GGCTCCCGCATCCAGAAGAGAGCCTGCAACACTGCCACCTGCATGACCCATCGCTTGGCA \begin{tabular}{llllllllllllllllllll}
$G$ & $S$ & $R$ & $I$ & $Q$ & $\underline{\underline{K}}$ & $R$ & $A$ & $C$ & $N$ & $T$ & $A$ & $T$ & $C$ & $M$ & $T$ & $H$ & $R$ & $L$ & $A$ \\
\hline
\end{tabular} GGCTGGCTGAGCAGATCTGGGAGTATGGTGAGGAGCAACTTGCTGCCGACCAAGATGGGT \begin{tabular}{llllllllllllllllllllll}
\hline G & W & L & S & R & S & G & S & M & V & R & S & N & L & L & P & T & K & M & G \\
\hline
\end{tabular} TTCAAGATCTTCAGTGGGCCCCGCAGGAACTTCTGGTTTTAAGCCGTGAAATGATTATTG \begin{tabular}{lllllllllllllllll}
\hline $\mathrm{F}$ & $\mathrm{K}$ & $\mathrm{I}$ & $\mathrm{F}$ & $\mathrm{S}$ & $\mathrm{G}$ & $\mathrm{P}$ & $\underline{\underline{R}}$ & $\underline{R}$ & $N$ & $F$ & $W$ & $F$ & $*$
\end{tabular}

GAATAAGGTCCCCAGGAAGCTGAACTCTACTTTTAGTTTGCAATGAAGGCATCTTACAAA AAAAGAAAATAGCATGGAAGATACCCATGTATGCATGCTTCTCAATATTGAAAACATTCT TCTTTTCCTTGAAATAAAGTAAATAATTAAAGTTAAAAAAAAA

B

TGCTCCCAGCCACCTGGTACCGGCTGTCAGAGAGGTGTCATGGGGTTCTGGAAGTTCCCC $\begin{array}{lllllllllll}M & G & F & W & K & F & P\end{array}$ CCGTTCTTGGTCCTCAGCATCCTGGTCTTGTACCAGGCGGGCATGTTTCATGCAGCACCA $\begin{array}{lllllllllllllllllllll}P & F & L & V & L & S & I & L & V & L & Y & \& & A & G & M & F & H & A & A & \mathrm{P}\end{array}$ TTCAGGTCTGTCTTTGATGGGCGTTTTGATCCTGCTACCCTGGATGAGGAGGAATCGCGC $\begin{array}{llllllllllllllllllll}F & R & S & V & F & D & G & R & F & D & P & A & T & L & D & E & E & E & S & R\end{array}$ CTCCTACTGGCTGCGATGGTGAATGACTACGAGCAGATGAGGGCCCGGGAGTCGGAGAAG $\begin{array}{llllllllllllllllllll}\text { L } & L & L & A & A & M & \text { V } & \text { N } & \text { D } & \text { Y } & E & Q & \text { M } & R & A & R & E & S & E & K\end{array}$ GCTCAGAAGACCGAGGGCTCCCGCATCCAGAAGAGAGCCTGCAATACTGCCACCTGCATG \begin{tabular}{lllllllllllllllllllll}
$A$ & $Q$ & $K$ & $T$ & $E$ & $G$ & $S$ & $R$ & $I$ & $Q$ & $K$ & $R$ & $A$ & $C$ & $N$ & $T$ & $A$ & $T$ & $C$ & $M$ \\
\hline
\end{tabular} ACCCATCGCTTGGCAGGCTGGCTGAGCAGGTCTGGGAGTATGGTGAGGAGCAACTTGCTG \begin{tabular}{lllllllllllllllllllll}
\hline $\mathrm{T}$ & $\mathrm{H}$ & $\mathrm{R}$ & $\mathrm{L}$ & $\mathrm{A}$ & $\mathrm{G}$ & $\mathrm{W}$ & $\mathrm{L}$ & $\mathrm{S}$ & $\mathrm{R}$ & $\mathrm{S}$ & $\mathrm{G}$ & $\mathrm{S}$ & $\mathrm{M}$ & $\mathrm{V}$ & $\mathrm{R}$ & $\mathrm{S}$ & $\mathrm{N}$ & $\mathrm{L}$ & $\mathrm{L}$ \\
\hline
\end{tabular} CCGACCAAGATGGGTTTCAAGATCTTCAGTGGGCCCCGCAAGAACTTCTGGTTTTAAGCC \begin{tabular}{llllllllllllllllllll}
\hline $\mathrm{P}$ & $\mathrm{T}$ & $\mathrm{K}$ & $\mathrm{M}$ & $\mathrm{G}$ & $\mathrm{F}$ & $\mathrm{K}$ & $\mathrm{I}$ & $\mathrm{F}$ & $\mathrm{S}$ & $\mathrm{G}$ & $\mathrm{P}$ & $\mathrm{R}$ & $\mathrm{K}$ & $\mathrm{N}$ & $\mathrm{F}$ & $\mathrm{W}$ & $\mathrm{F}$ & * \\
\hline
\end{tabular}

GTGAAATGATTATTGGAATAAGGTCCCCAGGAAGCTGAACTCTACTTTTAGTTTGCAATG AAGGCATCTTACAAAAAAAGAAAATAGCATGGAAGATACCCATGTATGCATGCTTCTCGA TATTGAAAACATTCTTCTTTTCCTTGAAATAAAGTAAATGCAGAATAAAAAAG

C CCCAGTTCCTGCTGCCAGAGAGGCGTCATGGGGTTCTGGAAGTTTCTGCCCTTCCTGGTC $\begin{array}{lllllllllllllll}M & G & F & W & K & F & L & P & F & L & V\end{array}$ CTCAGCTTCCTGGTCGTGTACCAGGCAGGCATGTTCCAGGCGGCACCATTCAGGTCAGCC $\begin{array}{llllllllllllllllllll}L & S & F & L & V & V & Y & Q & A & G & M & F & Q & A & A & P & F & R & S & A\end{array}$ TTGGAGAATGACTTTGATCCTGCTATACTCACAGAGAAGGAAATGTGCCTCCTACTGGCC $\begin{array}{lllllllllllllllllllll}L & E & N & D & F & D & P & A & I & L & T & E & K & E & M & C & L & L & L & A\end{array}$ GCAGTGATGAACGATTATGTGCAGATGAAGACCAGTGAACTGAAGCAGGAGGCAGAGCAC

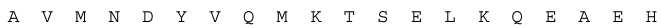
TTCCACATCACTGCCCAGAAAAGATCCTGTAACAGGGCCACCTGTGTGACCCATAAGATC

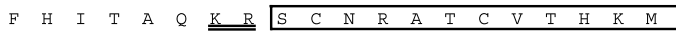
GCAGGCTCACTAAGCAGATCTGGGAGTGAGATTAAGAGGAACTTCATGTCTACTAATGTG \begin{tabular}{llllllllllllllllllllll}
\hline$A$ & $G$ & $S$ & $L$ & $S$ & $R$ & $S$ & $G$ & $S$ & $E$ & I & $K$ & $R$ & N & F & M & S & T & N & V \\
\hline
\end{tabular} GGCTCCAAAGCCTTTGGCCAGCGCAGCAGGGACCTTCAGAAAATGAGCAGTGAAATGACT \begin{tabular}{lllllll|lllllllllllllll}
\hline$G$ & $S$ & $K$ & $A$ & $F$ & $G$ & $Q$ & $R$ & $S$ & $R$ & $D$ & $L$ & $Q$ & $K$ & $M$ & $S$ & $S$ & $E$ & $M$ & $T$
\end{tabular} CCAGGAAGAAAGGTCACCAAGAAGCTGAACTCCATTTCTTTTAATTTGCATGAAACTAAC

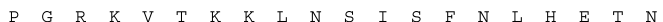
TTATTGAAAAAAAATTGAATGGAAGATACACATATATGCATGCCTCTTGATACTGAAAAC L L K K $\quad \mathrm{N}$

ATTTCTTTTTGCTTGAAATAAAGGCAAATGCAGAATAAAATCATTCGATATC

Figure 1 Nucleotide and deduced amino acid sequences of (A) ovine CRSP-1 precursor, (B) caprine CRSP-1 precursor, and (C) caprine CRSP-2 precursor. Nucleotide and amino acid numbers are shown on the right. Deduced mature amino acid sequences of ovine and caprine CRSP-1, and caprine CRSP-2 are boxed. The termination codons are marked with asterisk. Putative prohormone convertase cleavage sites are double underlined, and putative signal sequences are shown in italics. of the study. Repeated-measures ANOVA is appropriate as it simultaneously incorporates the time and treatment (within animal) effects without direct recourse to numerous pairwise comparisons, and is thus routinely used in this context with group sizes of five to eight using similar experimental design (Charles et al. 1990, 1991, 2001, 2006). The appropriateness of the parametric analysis has been confirmed with attention to graphical displays of the residuals from the models confirming appropriate normality. Statistical significance was assumed at $P<0 \cdot 05$. Where significant differences were identified by ANOVA, a priori Fisher's protected least-squares difference (LSD) tests were used to identify time points significantly different from time-matched control. Fisher's LSD in this context is a corrected pairwise comparison involving within-animal effects.

\section{Results}

Amino acid sequences of ovine CRSP-1 and caprine CRSP-1 and CRSP-2

To determine amino acid sequences of ovine and caprine CRSP precursors, ovine and caprine cDNA libraries were constructed, and were used for screening with ${ }^{32} \mathrm{P}-$ labeled porcine CRSP-1 cDNA as a probe. Out of $3 \times 10^{5}$ clones of ovine and caprine cDNA libraries respectively, 35 and 14 positive clones were obtained. By nucleotide sequence analysis, all the 35 ovine positive clones were shown to have an identical sequence. Among the 14 caprine positive clones, 9 clones encoded one identical sequence, while the other 5 clones encoded a second sequence. Based on the similarity in amino acid sequences deduced from the nucleotide sequences along with biological activities, as described below, the ovine clone and one of the caprine clones were categorized as CRSP-1, while the other caprine clone was categorized as CRSP-2. The nucleotide sequences and their deduced amino acid sequences are shown in Fig. 1. All CRSPs identified in this

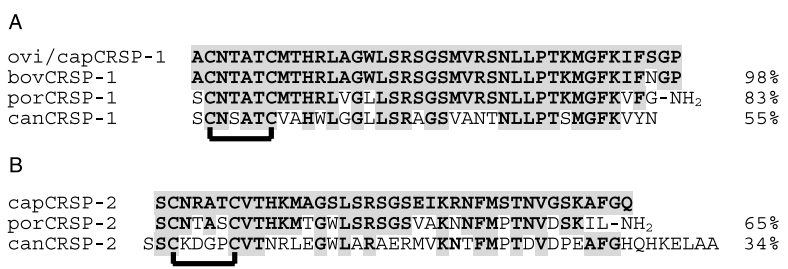

Figure 2 Comparison of amino acid sequences of ovine/caprine CRSP-1 and caprine CRSP-2 with corresponding peptides in other animals. (A) The amino acid sequence of ovine/caprine CRSP-1 (ovi/capCRSP-1) is aligned with those of bovine CRSP-1 (bovCRSP-1), porcine CRSP-1 (porCRSP-1), and canine CRSP-1 (canCRSP-1). (B) The amino acid sequence of caprine CRSP-2 (capCRSP-2) is aligned with those of porcine CRSP-2 (porCRSP-2) and canine CRSP-2 (canCRSP-2). Identical residues to (A) ovine/caprine CRSP-1 or (B) caprine CRSP-2 are shown in boldface and shaded, and the percentage of amino acid sequence identity to (A) ovine/caprine CRSP-1 or (B) caprine CRSP-2 is shown on the right. Brackets show the disulfide linkage. 

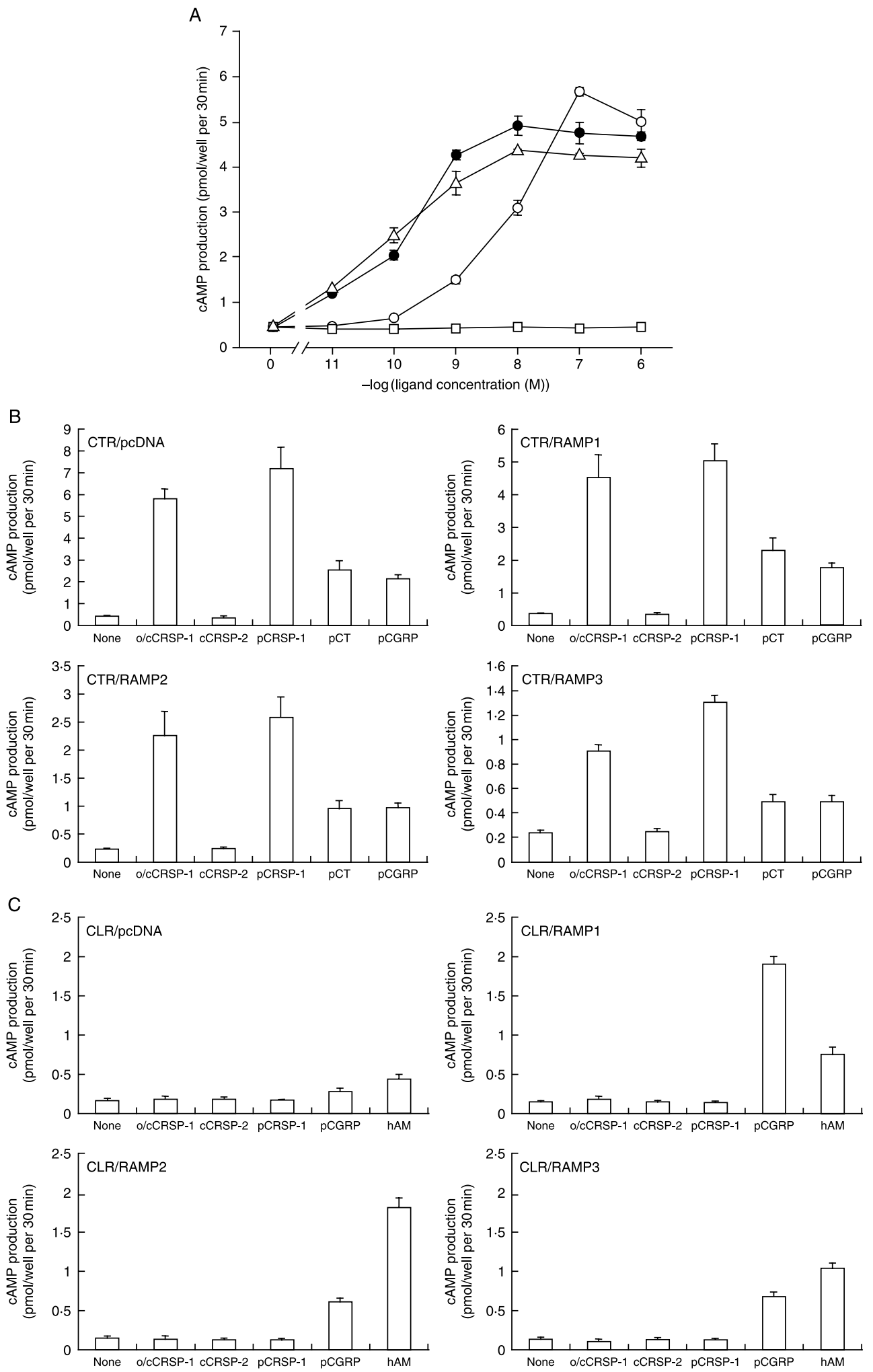
study have typical features of peptides in the CGRP family with a signal sequence and $\mathrm{N}$-terminal flanking peptide, but all sequences lack a donor glycine for the C-terminal amidation which exists in the members of CGRP family, in between mature peptide and C-terminal flanking peptide.

Figure 2 shows the comparison of amino acid sequences of mature ovine and caprine CRSP-1, and caprine CRSP-2 with CRSP-1 and CRSP-2 peptides in other species known, to date. Amino acid sequences of mature ovine and caprine CRSP-1 are identical, and have only one amino acid substitution compared with that of bovine CRSP-1. The amino acid sequence of ovine/caprine CRSP-1 also shows high identity (83\%) with porcine CRSP-1. Ovine/caprine CRSP-1 shows less amino acid sequence homology (55\%) with canine CRSP-1. In contrast to CRSP-1, the CRSP-2 peptides show much less degree of amino acid sequence conservation. The highest amino acid sequence identity is observed between caprine and porcine CRSP-2 (65\%) with amino acid sequence identity between caprine and canine CRSP-2 being only $34 \%$.

\section{Effects of CRSPs on Recombinant CT-R and CL-R}

Using synthetic peptides of ovine/caprine CRSP-1 and caprine CRSP-2, we first evaluated effects of CRSPs on porcine CT-R, which has strong affinity to porcine CRSP-1 and mediates its signal into cells by activating adenylyl cyclase. Porcine CT-R was transiently expressed in COS-7 cells, and then those cells were stimulated with ovine/caprine CRSP-1 or caprine CRSP-2 and compared with stimulation with porcine CRSP-1 and CT. As shown in Fig. 3A, all CRSP-1 peptides and porcine CT stimulated cAMP production in a dose-dependent manner. By contrast, caprine CRSP-2 did not alter cAMP production at all, results similar to those seen with CRSP-2 peptides from other species such as pig and dog. The potency of ovine/caprine CRSP-1 in the porcine CT-R activation was almost as strong as that of porcine CRSP-1, and much stronger than that of porcine CT. We then evaluated the effect of CRSPs on CT-RRAMP or CL-R-RAMP co-expression systems to observe the effect of those peptides on CT, CGRP, or AM receptors. As described by McLatchie et al. (1998), CL-R forms CGRP receptor when it is co-expressed with RAMP1, and forms AM receptor when it is co-expressed with RAMP2 or RAMP3. Figure $3 \mathrm{~B}$ and $\mathrm{C}$ shows the effect of the CRSPs, CT, CGRP, and AM, on cAMP production in cells that were transiently expressing CT-R or CL-R either alone or with one of three RAMPs. These peptides did not alter cAMP level in COS-7 cells with pcDNA3.1 empty vector (data not shown). Ovine/ caprine CRSP-1 stimulated cAMP production from cells co-expressing CT-R and the RAMPs, no matter which RAMP was co-expressed. However, none of the CRSPs stimulated cells expressing CL-R with or without any of RAMPs. These results indicate that ovine/caprine CRSP1 stimulates CT-R with potency similar to that of porcine CRSP-1 but does not stimulate CGRP or AM receptor, and that CRSP-2 does not stimulate either CT-R or CL-R at all, even in the presence of any RAMPs.

\section{Bioactivity of ovine CRSP-1 in conscious sheep}

Experiments were completed without mishap and data collection was complete. There was no significant difference in baseline levels of most variables between study days apart from plasma corrected Ca that was lower at baseline $(-30$ and 0 min) on the day CRSP-1 was infused compared with control day $(2 \cdot 52 \pm 0 \cdot 054$ vs $2 \cdot 59 \pm 0 \cdot 028, P<0 \cdot 01)$. Therefore, plasma corrected $\mathrm{Ca}$ data are presented as percentage change from baseline.

Infusion of CRSP-1 induced significant decreases in plasma total $\mathrm{Ca}(P=0 \cdot 02)$ and corrected Ca levels $(P=0 \cdot 017)$ (Fig. 4). Reduction in Ca was apparent at both doses of CRSP- 1 and the levels remained lower than time-matched control levels for at least $60 \mathrm{~min}$ following the cessation of CRSP-1 infusions. Plasma ionized Ca tended to be lower throughout the infusion of CRSP-1 but these changes did not achieve statistical significance (Fig. 4). CRSP-1 infusions induced dose-dependent increases in plasma cAMP $(P=0 \cdot 002)$ with levels being elevated twofold over time-matched control by the end of the higher dose of CRSP-1 and levels returning toward control by $60 \mathrm{~min}$ post-infusion (Fig. 4).

CRSP-1 infusions at both doses had no significant effect on hemodynamic indices measured including MAP, heart rate, RAP cardiac output, and hematocrit (Fig. 5). Similarly, CRSP-1 infusions did not change plasma catecholamines (Fig. 6). Despite plasma aldosterone levels appearing to be lower than time-matched control data throughout the CRSP infusions, these differences did not achieve statistical significance $(P=0.36)$ (Fig. 6). However, there were subtle but significant effects of CRSP-1 observed on both plasma NT-CNP $(P=0.006)$ and PRA $(P=0.028)$ (Fig. 6). In the case of NT-CNP, Fisher's LSD testing showed that plasma NT-CNP levels were lower than time-matched control on the CRSP day at one time point during both the baseline period $(-30)$ and the

Figure 3 Effects of ovine/caprine CRSP-1, caprine CRSP-2, and its related peptides on cAMP production in COS-7 cells expressing CT-R alone, or CT-R or CL-R with RAMPs. (A) Dose-dependent stimulatory effects of CRSPs on adenylyl cyclase activity via porcine CT-R. COS-7 cells expressing porcine CT-R were stimulated with the indicated concentrations of ovine/caprine CRSP-1 ( $)$ ), caprine CRSP-2 ( $\square$ ), porcine CRSP-1 $(\triangle)$, or porcine CT $(O)$. Effects of CRSPs on cAMP production via porcine (B) CT-R or $(C)$ CL-R in the presence or absence of RAMPs. COS-7 cells were co-transfected with porcine CT-R or CL-R CDNA and one of porcine RAMP1, 2, 3 CDNA, or pcDNA using Lipofectamine LTX with Plus reagent, cultured for $24 \mathrm{~h}$, and then used for the experiments. The cells were stimulated with $100 \mathrm{nM}$ of each peptide. o, ovine; c, caprine; p, porcine; $h$, human. Each point represents the mean \pm s.D. of (A) three and (B and C) four separate determinations. 


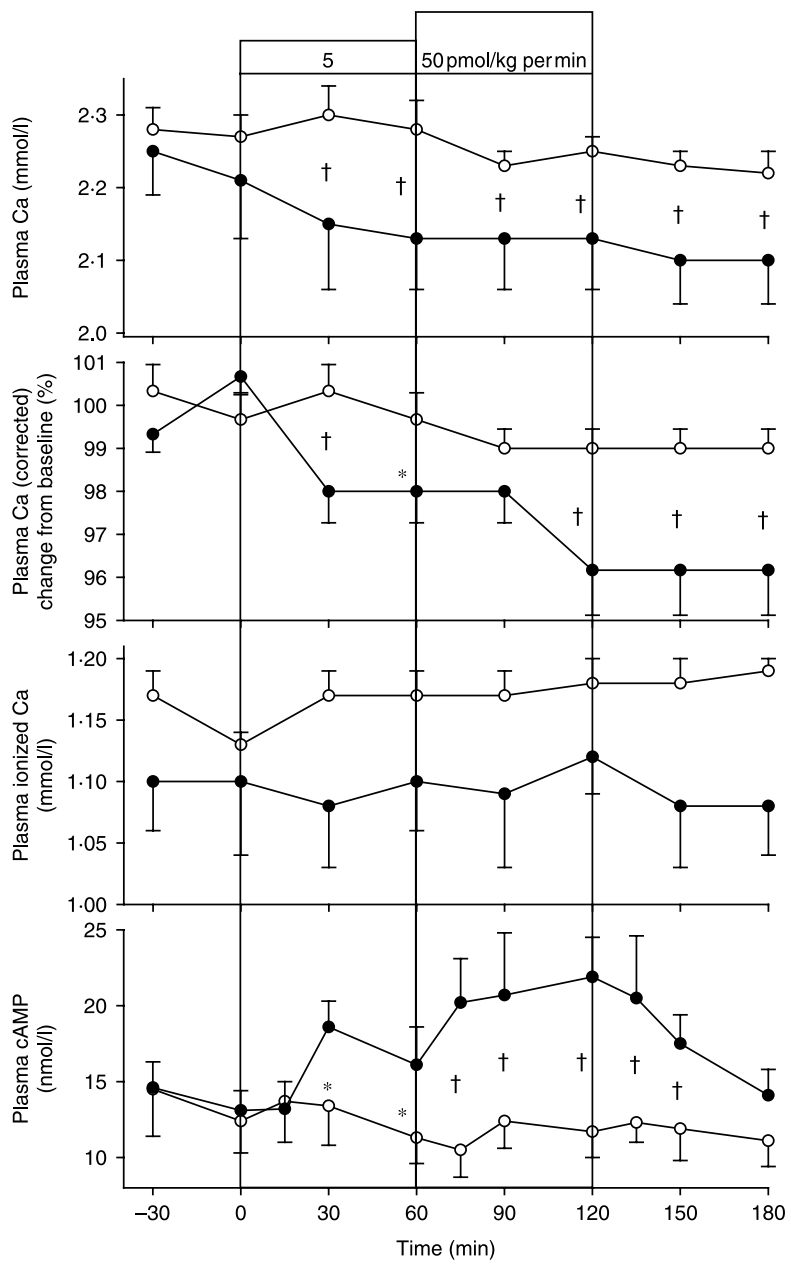

Figure 4 Plasma total, corrected, and ionized calcium (Ca) and cAMP response to i.v. infusions of CRSP-1 (-) or vehicle control $(O)$ in six sheep. Values shown are mean \pm s.E.M. Significant differences were observed for plasma total $\mathrm{Ca}(P=0 \cdot 02)$, corrected Ca $(P=0 \cdot 017)$, and $\mathrm{CAMP}(P=0 \cdot 002)$. Individual time points significantly different from time-matched control (Fisher's protected LSD) are indicated by ${ }^{*} P<0 \cdot 05$ and ${ }^{\dagger} P<0 \cdot 01$.

CRSP infusions (60 min). However, on cessation of the infusions, there was significant rebound activation of levels above control levels. Similarly, PRA showed a small but significant reduction at one time point $(75 \mathrm{~min})$ during CRSP infusion followed by significant rebound activation of levels above control levels on cessation of the CRSP-1 infusion. Taken together, these results demonstrating 'rebound activation' are indicative of relative suppression during the infusion of CRSP balanced or masked by counter-regulatory actions to stimulate NT-CNP and PRA levels, which, on termination of the infusion, result in a rise in these levels.

Urinary Ca excretion tended to be lower during the CRSP-1 arm of the study, but this increased output was apparent before the first dose of CRSP-1 (Fig. 7). Therefore, administration of CRSP-1 at both doses had no significant effect on urinary volume, sodium, potassium, or Ca excretion.

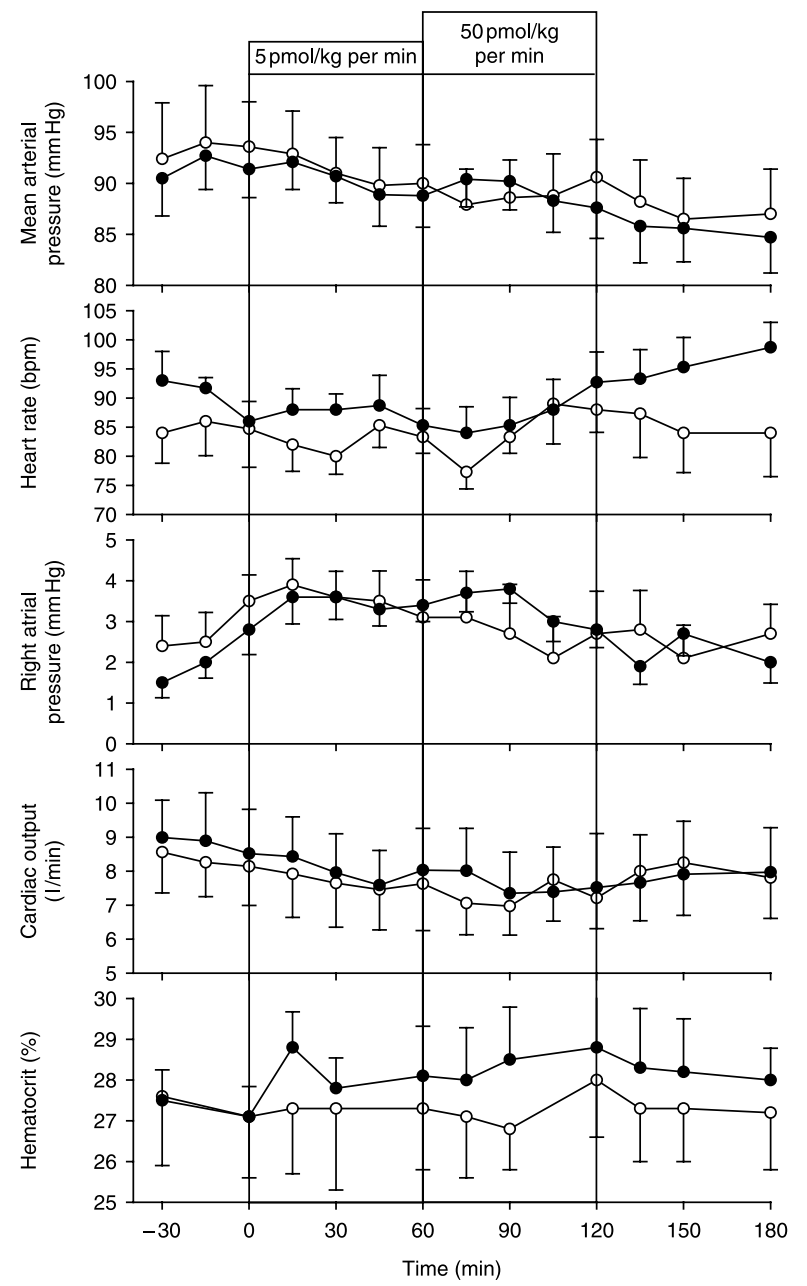

Figure 5 Hemodynamic response to i.v. infusions of CRSP-1 ( $)$ or vehicle control $(O)$ in six sheep. Values shown are mean \pm s.E.M.

\section{Discussion}

CRSP-1 is a member of the CGRP family and has been isolated in a number of mammalian species including pig, cow, dog, and horse (Katafuchi et al. 2003a, 2004). Studies to date indicate that CRSP-1 elicits biological activity, including elevation of CAMP, through the CT-R in vitro. Limited in vivo data show that porcine CRSP-1 lowers plasma Ca when injected into anesthetized rats but does not alter blood pressure. To date, there have been no integrated bioactivity studies of CRSP-1 performed in a large conscious animal model. Furthermore, there have been no in vivo studies of the biological actions of a CRSP-1 peptide administered to the species of origin. We now report the amino acid sequence and in vitro characterization of ovine/caprine CRSP-1 and caprine CRSP-2. Furthermore, we have examined the hemodynamic, electrolyte, hormonal, and renal effects of CRSP-1 administered as i.v. infusions to normal conscious sheep. CRSP-1 lowered plasma Ca with effects still apparent 


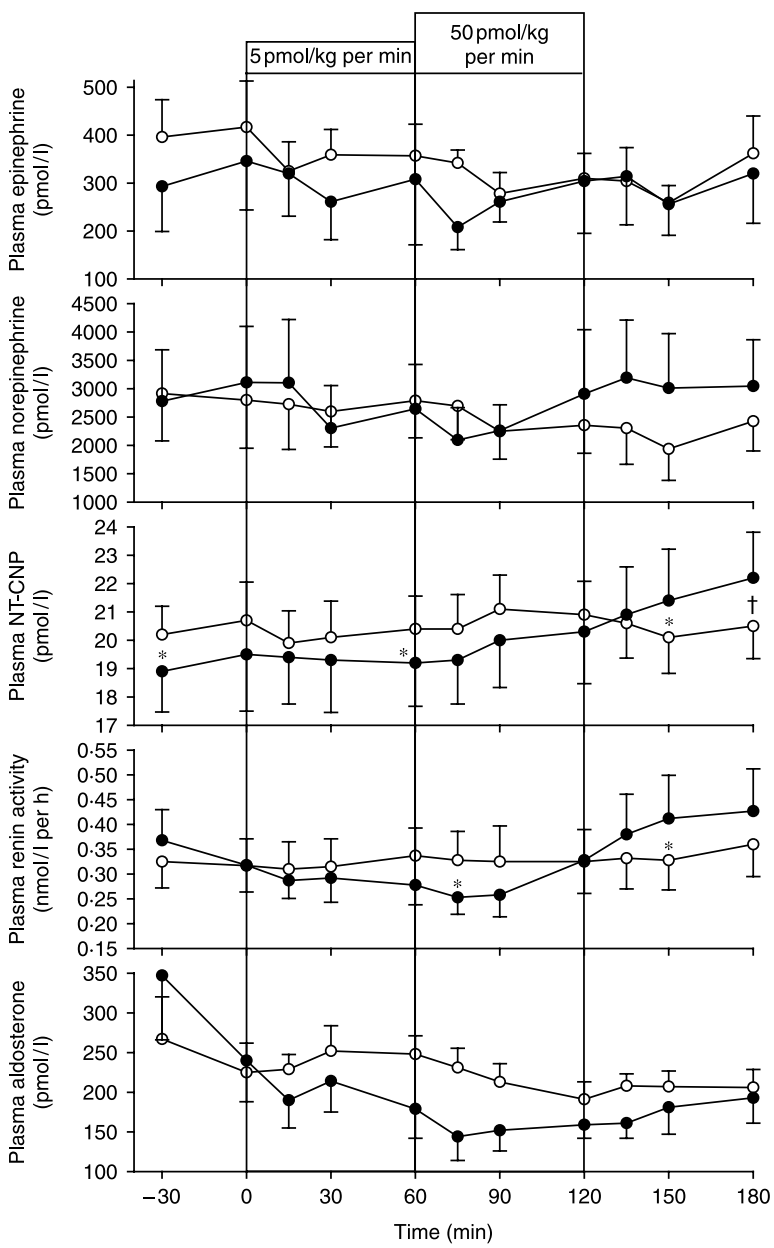

Figure 6 Plasma hormone response to i.v. infusions of CRSP-1 ( or vehicle control $(O)$ in six sheep. Values shown are mean \pm s.E.M. Significant differences were observed for plasma amino-terminal pro C-type natriuretic peptide (NT-CNP) $(P=0.006)$ and plasma renin activity $(P=0 \cdot 028)$. Individual time points significantly different from time-matched control (Fisher's protected LSD) are indicated by ${ }^{*} P<0 \cdot 05$ and ${ }^{+} P<0 \cdot 01$.

60 min following cessation of infusion. CRSP-1 infusions also resulted in significant dose-dependent rises in plasma cAMP. Both plasma NT-CNP and PRA were reduced during CRSP-1 infusions and showed rebound activation above time-matched control levels post-infusion. There were no significant effects observed on any hemodynamic and renal indices or other hormones measured.

The cDNA and deduced amino acid sequences of ovine and caprine CRSP-1 are shown in Fig. 1A and B. In evolutionarily closely related animals, amino acid sequences of CRSP-1 peptides are similar to each other. Deduced mature amino acid sequences of ovine and caprine CRSP-1 are identical to each other, almost identical to that of bovine CRSP-1 peptide, and show $83 \%$ identity with that of porcine CRSP-1. Like canine and bovine CRSP-1, ovine/caprine CRSP-1 does not have a $\mathrm{C}$-terminal amide that exists in other members of the CGRP

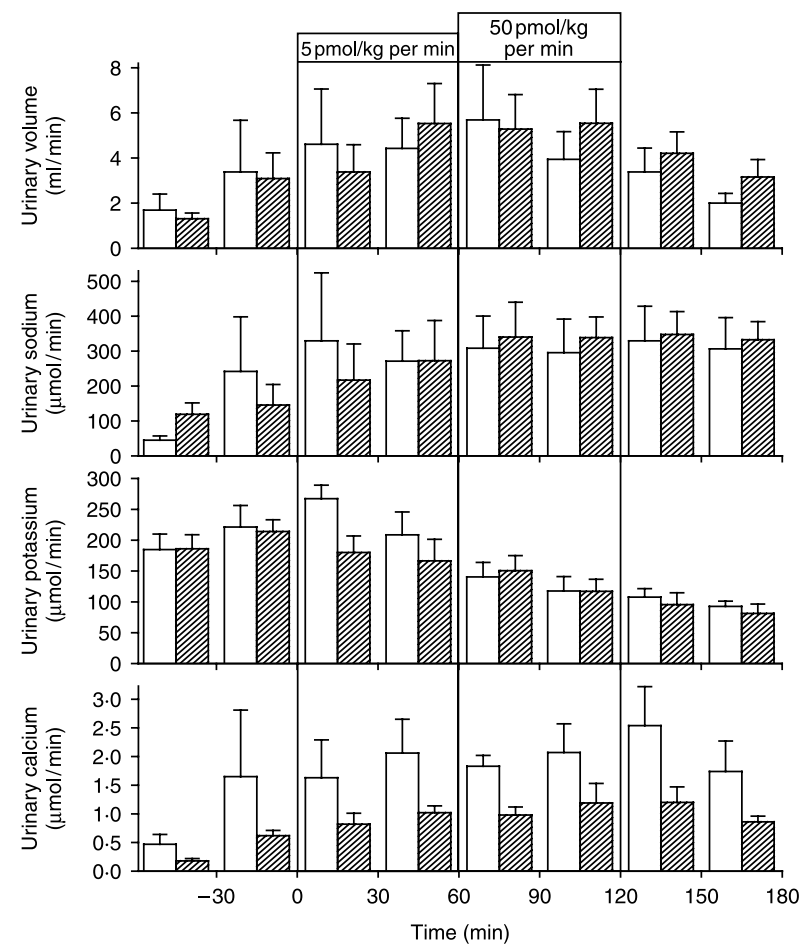

Figure 7 Renal response to i.v. infusions of CRSP-1 (hatched bars) or vehicle control (open bars) in six sheep. Values shown are mean \pm S.E.M.

family. Despite having the non-amidated C-terminus, ovine/ caprine CRSP-1 potently stimulated CT-R in a manner similar to porcine CRSP-1. Of note, the C-terminal glycine extended form of porcine CRSP-1 has been shown to be just as potent in stimulating CT-R as CRSP-1 (Katafuchi et al. 2005), thus supporting the idea that the C-terminal sequence and/or amidation are not essential for the interaction between CRSP-1 and its receptor, in contrast to other members of the CGRP family. Neither CT nor CRSP-1 stimulated CGRP receptor and AM receptor composed of CL-R and RAMPs, both of which require the C-terminal amidation of ligand peptides. The expression of any RAMP did not alter the specific stimulation of CT-R by CRSP-1, regardless of their differences in the sequence and C-terminal amidation. Taken together, ovine and caprine CRSP- 1 are confirmed to share the structural and biological features of CRSP-1 so far identified. By contrast, caprine CRSP-2 did not stimulate either CT-R or CL-R, even in the presence of any of RAMPs. Similarly, neither porcine nor canine CRSP-2 stimulated any combination of these receptors and RAMPs. Unlike precursors for porcine and canine CRSP-2 peptides that have no typical prohormone convertase processing site on either end of the deduced mature peptides, the precursor of caprine CRSP-2 has a typical prohormone convertase processing site between the $\mathrm{N}$-terminal flanking peptide and the deduced mature CRSP-2. Nevertheless, the synthetic CRSP-2 peptides used for this and previous studies might not be endogenous forms of CRSP-2 peptides (Katafuchi et al. 2003b). 
Purification and structural determination of native CRSP-2 peptides is a prerequisite for evaluating the function and biological activity of CRSP-2 peptides.

The existence of CT, a peptide that lowers plasma $\mathrm{Ca}$ and potently inhibits bone resorption, has been known for some 40 years (Zaida et al. 2002). We have recently identified a series of CRSP-1 peptides in mammalian species, which more potently activate the CT-R in vitro (Katafuchi et al. $2003 a, b$, 2004). Early in vivo studies showed that porcine CRSP-1 lowers plasma Ca when administered to anaesthetized rats (Katafuchi et al. 2003a). The present study extends this finding by demonstrating that ovine CRSP-1 administered to its species of origin exhibits potent, long-lasting actions to lower plasma Ca levels, presumably by activation of the CT-R. It is likely that CRSP-1 acts via stimulation of cAMP as circulating levels of cAMP showed a dosedependent rise to approximately twofold time-matched control levels during the high-dose infusion. This is consistent with potent stimulation of cAMP production observed by the CRSP-1 peptides in vitro both in the present study and in the previous reports (Katafuchi et al. 2003a,b, 2004). CRSP-1 has been previously shown to dose dependently inhibit the formation and activity of osteoclast-like cells in vitro (Notoya et al. 2007). Of interest in the present study, plasma levels of NT-CNP were subtly but significantly suppressed during CRSP-1 infusion with rebound activation above timematched control post-infusion. NT-CNP is strongly correlated with skeletal growth and markers of bone formation and as such is an emerging marker of growth plate activity in humans and other mammals (Prickett et al. 2005). Taken together, these results suggest that CRSP-1 is likely to be involved in bone turnover by inhibition of osteoclast formation and activity.

As stated earlier, CRSP-1 is a member of the CGRP family that includes CT, CGRP, and AM. Given the sequence homology between peptides in this family, it is of interest to speculate whether CRSP-1 may activate not only the CT-R but also the CL-R-RAMP complexes. CGRP activates CL-R-RAMP1 while AM and AM-2 activate the CL-RRAMP2 and CL-R-RAMP3 receptor complexes. Activation of these receptor complexes by CGRP (Gnaedinger et al. 1989), AM (Charles et al. 2001), and AM-2 (Charles et al. 2006) results in a number of hemodynamic actions including lowering blood pressure and peripheral resistance while increasing heart rate and cardiac output. These changes are also associated with significant activation of PRA. Of note in the present study, CRSP-1 had no effect on any of these hemodynamic indices and actually lowered PRA. Thus, results of this in vivo study suggest that CRSP-1, at least at these doses in sheep, does not bind to and/or signal via CL-R-RAMP receptor complexes. This is in agreement with the in vitro studies of this and previous studies which show that the CRSPs appear not to be ligands for any of the combinations of the CL-R-RAMP complexes (Katafuchi et al. 2003a,b, 2004). This is the first report of an effect of
CRSP-1 on PRA, but the significance of this finding requires further studies.

While reduction of plasma $\mathrm{Ca}$ is the major biological effect attributable to CT, administration of CT to men has also been shown to induce a number of renal effects including increased urinary volume, cAMP, electrolyte excretion, and glomerular filtration (Gnaedinger et al. 1989). This study showed no evidence for such renal effects of CRSP-1, at least at these doses in normal conscious sheep. In the rat, intracerebroventricular injection of porcine CRSP-1 is more potent than porcine $\mathrm{CT}$ in its effects on feeding and body temperature, whereas systemic administration of porcine CRSP-1 is less potent than porcine $\mathrm{CT}$ in reducing plasma $\mathrm{Ca}$ (Katafuchi et al. 2003a, Sawada et al. 2006). These results indicate that there might be two different states of CT-R: one that transduces a CRSP-1 signal more potently than CT, while the other transduces a CRSP-1 signal more weakly than CT. On the other hand, there are marked differences in the tissue expression level of CRSP-1 and CT in the central nervous system and thyroid gland. Therefore, it is necessary to take all these points into account when postulating a physiological role for CRSP-1.

In conclusion, we have identified ovine/caprine CRSP-1 and caprine CRSP-2. This newly identified CRSP-1 has been shown to share the structural and biological features of CRSP-1 peptides identified to date. In vivo studies confirm that CRSP-1 reduces plasma Ca levels, presumably via a cAMP-mediated mechanism, along with several new effects on the PRA and NT-CNP. By contrast, caprine CRSP-2 did not stimulate any combination of CT-R, CL-R, and RAMPs. Purification and structural determination of native CRSP-2 peptides is essential for searching and evaluating the function and biological activity of CRSP-2.

\section{Declaration of Interest}

The authors have no conflicts of interest to declare.

\section{Funding}

This work was supported in part by the Program for Promotion of Fundamental Studies in Health Sciences of the National Institute of Biomedical Innovation, Japan, and the Research Grant for Cardiovascular Disease from the Ministry of Health, Labor and Welfare of Japan. Support was also provided through grants from the National Heart Foundation of New Zealand and Health Research Council of New Zealand.

\section{Acknowledgements}

We are grateful to A Okabe for expert technical assistance, the staff of the University of Otago, Christchurch Animal Laboratory for assistance with animal studies, and Christchurch Cardioendocrine Laboratory staff for hormone assays. Caprine hypothalamus was kindly donated by Prof $\mathrm{M}$ Nakazato, Faculty of Medicine, University of Miyazaki. 


\section{References}

Charles CJ, Espiner EA, Cameron VA \& Richards AM 1990 Hemodynamic, renal, and endocrine actions of ANF in sheep: effect of 24-h, low-dose infusions. American Journal of Physiology 258 R1279-R1285.

Charles CJ, Espiner EA, Yandle TG, Cameron VA \& Richards AM 1991 Biological actions of cleaved atrial natriuretic factor (ANF101-105/106126) in conscious sheep. Journal of Cardiovascular Pharmacology 17 403-410.

Charles CJ, Nicholls MG, Rademaker MT \& Richards AM 2001

Comparative actions of adrenomedullin and nitroprusside: interactions with angiotensin II and norepinephrine. American Journal of Physiology 281 R1887-R1894.

Charles CJ, Rademaker MT \& Richards AM 2006 Hemodynamic, hormonal and renal actions of adrenomedullin-2 in normal conscious sheep. Endocrinology 147 1871-1877.

Dunn PJ \& Espiner EA 1976 Outpatient screening tests for primary aldosteronism. Australian and New Zealand Journal of Medicine 6 131-135.

Gnaedinger MP, Uehlinger DE, Weidmann P, Sha SG, Muff R, Born W, Rascher W \& Fischer JA 1989 Distinct hemodynamic and renal effects of calcitonin gene-related peptide and calcitonin in men. American Journal of Physiology 257 E848-E854.

Goldstein DS, Feuerstein G, Izzo JL, Kopin IJ \& Keiser HR 1981 Validity and reliability of liquid chromatography with electrochemical detection for measuring plasma levels of norepinephrine and epinephrine in man. Life Sciences 28 267-275.

Katafuchi T, Kikumoto K, Hamano K, Kangawa K, Matsuo H \& Minamino N $2003 a$ Calcitonin receptor-stimulating peptide, a new member of the calcitonin gene-related peptide family. Journal of Biological Chemistry 278 12046-12054.

Katafuchi T, Hamano K, Kikumoto K \& Minamino N $2003 b$ Identification of second and third calcitonin receptor-stimulating peptides in porcine brain. Biochemical and Biophysical Research Communications 308 445-451.

Katafuchi T, Hamano K \& Minamino N 2004 Identification, structural determination, and biological activity of bovine and canine calcitonin receptor-stimulating peptides. Biochemical and Biophysical Research Communications 313 74-79.
Katafuchi T, Hamano K, Kikumoto K \& Minamino N 2005 Isolation and characterization of a glycine-extended form of calcitonin receptorstimulating peptide-1: another biologically active form of calcitonin receptor-stimulating peptide-1. Peptides 26 2616-2623.

Lewis JG, Manley L, Whitlow JC \& Elder PA 1992 Production of a monoclonal antibody to cortisol: application to a direct immunosorbent assay of plasma. Steroids $\mathbf{5 7} 82-85$.

Lun S, Espiner EA, Nicholls MG \& Yandle TG 1983 A direct radioimmunoassay for aldosterone in plasma. Clinical Chemistry 29 268-271.

McLatchie LM, Fraser NJ, Main MJ, Wise A, Brown J, Thompson N, Solari R, Lee MG \& Foord SM 1998 RAMPs regulate the transport and ligand specificity of the calcitonin-receptor-like receptor. Nature 393 333-339.

Notoya M, Arai R, Katafuchi T, Minaminoa N \& Hagiwara H 2007 A novel member of the calcitonin gene-related peptide family, calcitonin receptorstimulating peptide, inhibits the formation and activity of osteoclasts. European Journal of Pharmacology $\mathbf{5 6 0}$ 234-239.

Prickett TCR, Yandle TG, Nicholls MG, Espiner EA \& Richards AM 2001 Identification of amino-terminal pro-C-type natriuretic peptide in human plasma. Biochemical and Biophysical Research Communications 286 513-517.

Prickett TCR, Lynn AM, Barrell GK, Darlow BA, Cameron VA, Espiner EA, Richards AM \& Yandle TG 2005 Amino-terminal pro-CNP: a putative marker of cartilage activity in postnatal growth. Pediatric Research $\mathbf{5 8}$ 334-340.

Sawada H, Yamaguchi H, Shimbara T, Toshinai K, Mondal MS, Date Y, Murakami N, Katafuchi T, Minamino N, Nunoi H et al. 2006 Central effects of calcitonin receptor-stimulating peptide-1 on energy homeostasis in rats. Endocrinology 147 2043-2050.

Zaida M, Inzerillo AM, Moonga BS, Bevis PJR \& Huang CL-H 2002 Forty years of calcitonin - where are we now? A tribute to the work of Iain Macintyre, FRS Bone 30 655-663.

Received in final form 11 May 2008

Accepted 15 May 2008

Made available online as an Accepted Preprint 15 May 2008 